Wir bieten:

3- und 5-jährige Ausbildung zur / zum
Psychologischen Psychotherapeutin/en

- Ausbildungsbeginn dreimal jährlich -

$O$ ärztliche Weiterbildung in Verhaltenstherapie

O Fortbildungsveranstaltungen

für Approbierte Psychotherapeuten

AVT GmbH • Venloer Straße 47-53 • 50672 Köln • www.avt-koeln.org Tel.: 0221 - 2585640 •Fax: 0221 - 25856420 • info@avt-koeln.org

\begin{tabular}{l}
\hline IVT-Kurpfalz gGmbH \\
Staatlich anerkanntes Ausbildungsinstitut \\
für Verhaltenstherapie \\
Augustaanlage 7-11·68165 Mannheim \\
Sekretariat: \\
Anette Krämer, Brigitte Unger \\
Mo-Fr 8.00 - 15.30 Uhr \\
Telefon: 0621 - 415364 \\
E-Mail: sekretariat@ivt-kurpfalz.de
\end{tabular}

Ausbildung in Kinderund Jugendlichen Psychotherapie mit Schwerpunkt Verhaltenstherapie

Staatlich anerkanntes Ausbildungsinstitut [Voraussetzung: Hochschulabschluss Pädagogik/Psychologie]

- Ausbildung in Kinder- und Jugendlichenpsychotherapie

- Qualifikation in Gruppenpsychotherapie

für Kinder, Jugendliche und Familien

- Einblick und Praktikumsmöglichkeit im Bereich Neurofeedback für ADHS-Behandlung im Rahmen der freien Spitze

- Individuelle Betreuung und Anleitung in der praktischen Ausbildung

- Große Institutsambulanz mit 16 Behandlungsräumen

- Möglichkeit im Lehrpraxenmodell zu arbeiten

27. August 2020 bis 31.08.2020: Gruppentherapie-Fortbildung Kinder-VT-Gruppe Abrechnungsgenehmigung Kinderverhaltenstherapi
in Gruppen, 50 Theoriestunden

\begin{tabular}{|c|}
$\begin{array}{c}27.08 .2020 \\
\text { bis } \\
31.08 .2020\end{array}$ \\
\hline
\end{tabular}

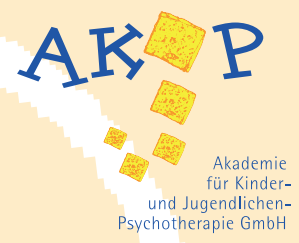

Kontakt: Dr. Josef Könning AKJP

Akademie

für Kinderund JugendlichenPsychotherapie GmbH Bohmter Str. 1 49074 Osnabrück

Tel. 0541-20227-91 Fax 0541-20227-96 akjp.gmbh@t-online.de WWW.kinderverhaltenstherapie.de 


\section{EMDR-Instifut Deutschland}

der autorisierte Partner des Instituts von

Francine Shapiro PhD, USA

\section{Zertifizierte EMDR-Ausbildung}

für Ärztl. und Psychol. Psychotherapeutlnnen und appr. KJP. Praxisbezogene Vermittlung unter Anleitung in Kleingruppen.

Gut erreichbare Seminarorte in Deutschland,

Österreich und Schweiz.

Info und Anmeldung:

www.emdr.de ·info@emdr.de ·Tel. +49 - (0) 2204 - 25866

\section{Institut für Fort- und Weiterbildung in klinischer Verhaltenstherapie e. V.}

Staatlich anerkanntes Ausbildungsinstitut

\section{Weiterbildungen}

$\rightarrow$ Schematherapie Kinder/Jugendliche (WS 1) am 18./19.10.2019

$\rightarrow$ Curriculum Traumatherapie (Fachkunde EMDR) Start: 25./26.10.2019

$\rightarrow$ Schematherapie Erwachsene (WS 1-3) vom 09.11.-13.11.2020

\section{Fortbildungstage}

$\rightarrow$ Achtsamkeitsbasierte kognitive Therapie für Kinder

und Jugendliche am 29.11.2019

$\rightarrow$ Kultursensible Traumatherapie mit Geflüchteten am 07.12.2019

$\rightarrow$ Kognitive Verfahren in der Verhaltenstherapie am 17./18.01.2020

Bewerbungen/Anmeldungen ab sofort möglich

Informationen und weitere Fortbildungsangebote:

FKV · Kurbrunnenstr. $21 \mathrm{a} \cdot 67098$ Bad Dürkheim

Tel. 06322 94828-0 - Fax: 06322 94828-29

E-mail: info@ifkv.de.Internet: www.ifkv.de

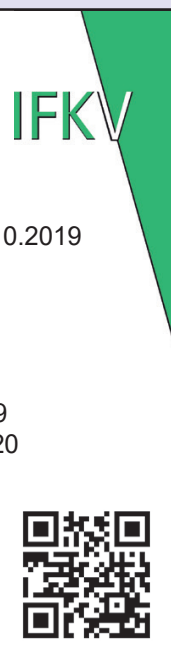

\section{FIKV FIKV - Fortbildungsinstitut für \\ Klinische Verhaltenstherapie}

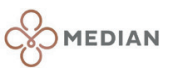

Staatlich anerkanntes Ausbildungsinstitut angegliedert an das MEDIAN Zentrum für Verhaltensmedizin Bad Pyrmont Wir bieten:

- 3-jährige Vollzeitausbildung zum/r Psychologischen Psychotherapeuten/in

- 3-jährige Tätigkeit auf entsprechend vergüteten Stellen als

«Postgraduierte Psychologen» in der Klinik

- Sämtliche Ausbildungsbausteine werden in unserer Klinik bzw. in unserem Ausbildungsinstitut durchgeführt.

- Die Kosten der Ausbildung tragen sich durch zusätzliche finanzielle

Unterstützung der Klinik und durch Einnahmen in der Ausbildungsambulanz.

- Ausbildungsbeginn jederzeit möglich

Information: $\quad$ FIKV, Bombergallee 11, 31812 Bad Pyrmont Tel. 05281 / 606763 oder 619620

sekretariat@fikv.de; www.fikv.de 